\title{
Randomized controlled trial: neostigmine for intra-abdominal hypertension in acute pancreatitis
}

\author{
Wenhua He ${ }^{1,2 \dagger}$, Peng Chen ${ }^{1 \dagger}$, Yupeng Lei ${ }^{1 \dagger}$, Liang Xia', Pi Liu' , Yong Zhu' ${ }^{1}$, Hao Zeng ${ }^{1}$, Yao Wu', Huajing Ke',
} Xin Huang ${ }^{1}$, Wenhao Cai ${ }^{2,3}$, Xin Sun ${ }^{4}$, Wei Huang ${ }^{3}$, Robert Sutton ${ }^{2}$, Yin Zhu ${ }^{1 *}$ and Nonghua Lu ${ }^{1 *}$ (D)

\begin{abstract}
Background: Intra-abdominal hypertension (IAH) in acute pancreatitis (AP) is associated with deterioration in organ function. This trial aimed to assess the efficacy of neostigmine for IAH in patients with AP.

Methods: In this single-center, randomized trial, consenting patients with IAH within 2 weeks of AP onset received conventional treatment for $24 \mathrm{~h}$. Patients with sustained intra-abdominal pressure (IAP) $\geq 12 \mathrm{mmHg}$ were randomized to receive intramuscular neostigmine ( $1 \mathrm{mg}$ every $12 \mathrm{~h}$ increased to every $8 \mathrm{~h}$ or every $6 \mathrm{~h}$, depending on response) or continue conventional treatment for 7 days. The primary outcome was the percent change of IAP at $24 \mathrm{~h}$ after randomization.

Results: A total of 80 patients were recruited to neostigmine $(n=40)$ or conventional treatment $(n=40)$. There was no significant difference in baseline parameters. The rate of decrease in IAP was significantly faster in the neostigmine group compared to the conventional group by $24 \mathrm{~h}$ (median with 25th-75th percentile: $-18.7 \%$ [ -28.4 to $-4.7 \%$ ] vs. $-5.4 \%[-18.0 \%$ to 0$], P=0.017$ ). This effect was more pronounced in patients with baseline IAP $\geq 15 \mathrm{mmHg}$ $(P=0.018)$. Per-protocol analysis confirmed these results $(P=0.03)$. Stool volume was consistently higher in the neostigmine group during the 7-day observational period (all $P<0.05$ ). Other secondary outcomes were not significantly different between neostigmine and conventional treatment groups.
\end{abstract}

Conclusion: Neostigmine reduced IAP and promoted defecation in patients with AP and IAH. These results warrant a larger, placebo-controlled, double-blind phase III trial.

Trial registration Clinical Trial No: NCT02543658 (registered August /27, 2015).

Keywords: Neostigmine, Acute pancreatitis, Intra-abdominal hypertension, Acute compartment syndrome

*Correspondence: zhuyin27@sina.com; lunonghua@ncu.edu.cn †Wenhua He, Peng Chen, and Yupeng Lei contributed equally to this work.

${ }^{1}$ Pancreatic Intensive Care Unit, Department of Gastroenterology, First Affiliated Hospital of Nanchang University, Nanchang, China

Full list of author information is available at the end of the article

\begin{abstract}
Introduction
Acute pancreatitis (AP) is a common disease of the digestive system [1]. Severe acute pancreatitis (SAP) with persistent organ failure is associated with an increased risk of death [2-5]. Intra-abdominal hypertension (IAH) is defined as a persistent increase in intra-abdominal pressure (IAP) $\geq 12 \mathrm{mmHg}$, according to the World Society for Abdominal Compartment Syndrome (WSACS) [6]. IAH is considered to be an early risk factor in the development of SAP [7]. In a prospective study, IAH was
\end{abstract}

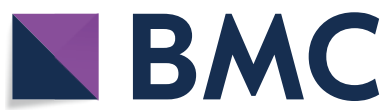

(c) The Author(s) 2022. Open Access This article is licensed under a Creative Commons Attribution 4.0 International License, which permits use, sharing, adaptation, distribution and reproduction in any medium or format, as long as you give appropriate credit to the original author(s) and the source, provide a link to the Creative Commons licence, and indicate if changes were made. The images or other third party material in this article are included in the article's Creative Commons licence, unless indicated otherwise in a credit line to the material. If material is not included in the article's Creative Commons licence and your intended use is not permitted by statutory regulation or exceeds the permitted use, you will need to obtain permission directly from the copyright holder. To view a copy of this licence, visit http://creativecommons.org/licenses/by/4.0/. The Creative Commons Public Domain Dedication waiver (http://creativeco mmons.org/publicdomain/zero/1.0/) applies to the data made available in this article, unless otherwise stated in a credit line to the data. 
diagnosed in $17 \%$ of patients with $\mathrm{AP}$, resulting in a mortality rate of $37 \%[8]$.

The inflammatory state of AP sparks a cascade of pancreatic and visceral edema, acute peripancreatic fluid collections, ascites, gut injury with paralytic ileus and gastric dilatation, leading to elevated IAP [9]. When IAP rises above $20 \mathrm{mmHg}$, abdominal compartment syndrome (ACS) and organ failure ensue [6]. AP complicated by ACS is associated with a mortality rate of 49\% [9]. Although surgical decompression can promptly improve ACS, it causes substantial morbidity [9]. Thus, non-operative strategies for reducing IAH in AP patients are preferred, including nasogastric decompression, promotility agents and percutaneous catheter drainage (PCD), etc. [6, 7]. Neostigmine is an anti-cholinesterase drug that can enhance intestinal peristalsis, promoting the passage of flatus and defecation. Treatment with neostigmine effectively induces colonic decompression among those patients with colonic pseudo-obstruction [10-14]. The WSACS has suggested neostigmine be used for the treatment of established colonic ileus associated with IAH that does not respond to other simple measures [6]. No robust evidence exists, however, on the effects of pharmacological promotility therapy for IAP or outcomes among those with IAH/ACS [6]. Whether neostigmine can effectively reduce IAP and is beneficial in AP is unclear. In addition, confirmation of colonic ileus requires an abdominal X-ray typically in a standing position or computed tomography (CT) of the abdomen, likely to be unsuitable for patients with organ failure or hemodynamical instability during the early phase of AP. Paralytic ileus is a common risk factor for IAH in patients with AP [7]. If conventional treatment fails to correct $\mathrm{IAH}$, there may be persistent paralytic ileus contributing to IAH, in which case neostigmine treatment may be beneficial.

This trial aimed to evaluate the efficacy of neostigmine in reducing IAP in patients with AP and persistent IAH following $24 \mathrm{~h}$ of conventional treatment, whether or not colonic pseudo-obstruction was established by X-ray or computerized tomography.

\section{Methods}

\section{Study design and participants}

This single-center, two-armed, parallel-group, superiority, randomized controlled phase II clinical trial was conducted between September 2015 and August 2017 in the Pancreatic Intensive Care Unit (ICU) of the Department of Gastroenterology at the First Affiliated Hospital of Nanchang University. This trial was registered (ClinicalTrials.gov, No. NCT02543658) and conducted adhering to a protocol that was approved by the Medical Ethics Research Committee of our hospital (No. 2015-011).

Patients aged between 18 and 70 years old who were within two weeks of AP onset and diagnosed with IAH during their Pancreatic ICU stay were assessed for eligibility. IAP was measured indirectly, using intravesicular pressure measured through a bladder catheter [6]. Briefly, the patient was in the supine position, $25 \mathrm{ml}$ sterile normal saline was injected through the bladder before measurement, and with the transducer zeroed at the level where the midaxillary line crosses the iliac crest. For mechanically ventilated patients, IAP was measured under sedation. For patients with spontaneous breathing, IAP was measured at end expiration and ensuring that abdominal muscle contractions are absent.

When IAP remained $\geq 12 \mathrm{mmHg}$ after conventional treatment (including sedation and analgesia, nasogastric decompression, glycerin enema for defecation, negative fluid balance and PCD for ascites) for $24 \mathrm{~h}$, participants were considered to be enrolled in the trial when they met no exclusion criteria. Exclusion criteria included: (1) history of laparotomy; (2) intra-abdominal bleeding; (3) contraindications to neostigmine: angina pectoris, myocardial infarction, ventricular tachycardia, bradycardia, acute circulatory failure, epilepsy, bronchial asthma, mechanical intestinal obstruction, hyperthyroidism, serious arrhythmia, intestinal fistula or allergy to neostigmine; (4) urinary tract infection, or previous bladder surgery; (5) pregnancy or lactation. All patients or their legal representatives provided written informed consent before randomization.

\section{Randomization and concealment}

Patients were enrolled in this trial by gastroenterologists who evaluated the study participants in the Pancreatic ICU. A statistician generated a randomization list with a computer program for use in sealed, opaque envelopes. The allocation sequence was concealed from the researchers. Once the patient was included in the study, the sealed envelope was opened by one of the study investigators to determine the treatment allocation. Participants were allocated to the neostigmine group or conventional group in a 1:1 ratio. As the dosing schedule of neostigmine depended on the response in an unpredictable manner, the clinicians, outcome assessors, and patients were not blinded from assignment to intervention.

\section{Intervention and follow-up}

In the neostigmine group, patients received an initial dose of neostigmine (given intramuscularly within minutes of randomization) of $1 \mathrm{mg}$ every $12 \mathrm{~h}$. If there was no defecation after $12 \mathrm{~h}$, the dose was increased to 
$1 \mathrm{mg}$ every $8 \mathrm{~h}$; if there was no defecation after $24 \mathrm{~h}$, the dose was increased to $1 \mathrm{mg}$ every $6 \mathrm{~h}$. Neostigmine was stopped if the IAP dropped below $12 \mathrm{mmHg}$; otherwise, it was administered continuously for 7 days. Both the neostigmine and conventional groups received concomitant treatments as follows: (1) gastrointestinal decompression with a nasogastric and/or rectal tube; (2) paraffin oil and liquid soaked preparation of rheum officinale (rhubarb) and glauber salt by a nasogastric or nasojejunal tube. These traditional Chinese medicine components are widely used in China to alleviate gut dysmotility and have been shown to mitigate the severity of AP in patients $[15,16]$; (3) glycerin enema to promote defecation; (4) PCD for ascites; (5) intravenous albumin, diuretics and when indicated renal replacement therapy for fluid overload; (6) sedation and analgesia to avoid agitation and patient-ventilator asynchrony.

Patients with IAP $<15 \mathrm{mmHg}$ received enteral nutrition (EN) through a nasojejunal tube. The rate was initiated at $20 \mathrm{ml} / \mathrm{h}$ and increased gradually by $15 \mathrm{ml}$ every $8 \mathrm{~h}$ to the goal rate $(25-35 \mathrm{kcal} / \mathrm{kg} / \mathrm{d})$, depending on patient tolerance $[17,18]$. EN was stopped temporarily when the IAP $\geq 15 \mathrm{mmHg}$ and parenteral nutrition was initiated. When any patient's IAP rose above $25 \mathrm{mmHg}$, or there were progressive organ dysfunction and fulminant
ACS [19], a multidisciplinary seminar was held, including gastroenterologists, surgeons, interventional physicians and intensive care physicians, to decide whether to perform a surgical decompression. All patients were followed-up at 1, 3 and 6 months after discharge through the outpatient interview or telephone connection. Patient demographics, hospitalization and follow-up data were recorded on standardized case record forms by an investigator or coordinator who was unaware of study-group assignments.

\section{Outcomes}

Definitions of the primary and secondary endpoints are displayed in Table 1. During the internal review process, we found neostigmine has the most significant effect in reducing IAP within $24 \mathrm{~h}$. Therefore, instead of the "percent change of IAP from randomization to 7 days" that registered in ClinicalTrials.gov., we modified the primary endpoint as "percent change of IAP at $24 \mathrm{~h}$ after randomization". We measured IAP at $3 \mathrm{~h}$ after randomization, then every $6 \mathrm{~h}$ for the following 3 days $(72 \mathrm{~h})$. After this period, IAP was measured as clinically indicated; patients who remained in Pancreatic ICU with IAP $\geq 12 \mathrm{mmHg}$ had IAP measured every $6 \mathrm{~h}$, while those transferred to general wards with normal IAP had IAP measured once

Table 1 Definition of the primary and secondary endpoints

\begin{tabular}{|c|c|}
\hline Endpoint & Definition \\
\hline $\mathrm{IAH}$ & A sustained or repeated pathological elevation in IAP $\geq 12 \mathrm{mmHg}$ \\
\hline \multirow[t]{4}{*}{ IAH grade } & Grade I, IAP 12-15 mmHg \\
\hline & Grade II, IAP 16-20 mmHg \\
\hline & Grade III, IAP 21-25 mmHg \\
\hline & Grade IV, IAP > 25 mmHg \\
\hline ACS & $\begin{array}{l}\text { A sustained IAP }>20 \mathrm{mmHg} \text { (with or without an APP }<60 \mathrm{mmHg} \text { ) that is associated with new organ dysfunction/ } \\
\text { failure }\end{array}$ \\
\hline Increase in stool volume & $\begin{array}{l}\text { Increase in } 24 \mathrm{~h} \text { stool volume on a designated day (day 1, day } 2 \text {, day } 3 \text {, day } 5 \text {, and day } 7 \text { ) after randomization above } \\
\text { the baseline } 24 \mathrm{~h} \text { stool volume before randomization }\end{array}$ \\
\hline New-onset ACS & ACS occurring after randomization (not present at any time before it), assessed for up to 4 weeks \\
\hline Deterioration of $\mathrm{IAH}$ & IAP that rebounds $\geq 5 \mathrm{mmHg}$ or increases to $\geq 20 \mathrm{mmHg}$ within 7 days after randomization \\
\hline New-onset organ failure & Organ failure occurring after randomization (not present at any time before randomization) \\
\hline Multiple-organ failure & Failure of two or more organs \\
\hline Respiratory failure & $\mathrm{PaO}_{2} / \mathrm{FiO}_{2} \leq 300$, or requirement for mechanical ventilation \\
\hline Circulatory failure & $\begin{array}{l}\text { Circulatory systolic blood pressure }<90 \mathrm{mmHg} \text {, despite adequate fluid resuscitation, or requirement for inotropic } \\
\text { catecholamine support }\end{array}$ \\
\hline Renal failure & Creatinine level > $177 \mu \mathrm{mol} / \mathrm{L}$ after rehydration or new need for haemofiltration or hemodialysis \\
\hline Timing of EN & Time from randomization to the initiation of tolerated EN \\
\hline Intra-abdominal bleeding & Intra-abdominal bleeding that requires surgical, radiologic, or endoscopic intervention \\
\hline Enterocutaneous or enteric fistula & $\begin{array}{l}\text { Secretion of fecal material from a percutaneous drain or inflow into a necrotic cavity, either from small or large } \\
\text { bowel, confirmed by endoscopy, imaging, or during surgery }\end{array}$ \\
\hline Adverse event & $\begin{array}{l}\text { The following events occurred during the use of neostigmine: drug eruption, ataxia, convulsions, coma, slurred } \\
\text { speech, anxiety, fear, cardiac arrest, or other untoward events not characteristic of or expected from AP; diarrhea } \\
\text { was excluded as this was part of the therapeutic effect to reduce IAP }\end{array}$ \\
\hline
\end{tabular}


every other day until 7 days after randomization. The following secondary endpoints were analyzed: (1) stool volumes at $24 \mathrm{~h}$ and 7 days after randomization; (2) timing of the start of EN; (3) deterioration of IAH; (4) new-onset ACS; (5) new-onset organ failure; (6) mortality for index hospital stay and within 6 months of follow-up; (7) other complications, adverse events and costs. Known local and systemic complications of AP, including new-onset organ failure occurring after neostigmine treatment or in the absence of neostigmine treatment, were not recorded as adverse events.

\section{Statistical analysis}

The sample size was calculated from our observational data in which IAP decreased by $30 \%$ after treatment with neostigmine for $24 \mathrm{~h}$ (10 patients), compared to $5 \%$ after conventional treatment for $24 \mathrm{~h}$ (10 patients) (unpublished data), predicting an absolute reduction in IAP of $25 \%$. Allowing for the possibility that neostigmine treatment might be better than conventional treatment and $10 \%$ loss to follow-up, we set the power at $80 \%$ and alpha at $5 \%$, requiring a sample size of 40 patients in each of the two groups, i.e., a total of 80 cases. Primary and secondary endpoints were compared between treatment groups, and both intention-to-treat and per-protocol analyses were performed. Furthermore, patients with baseline IAP above $15 \mathrm{mmHg}$ were selected for intention-to-treat subgroup analysis. Student's $t$-test was performed for continuous variables with normal distribution, and the Kruskal-Wallis $H$ test in the absence of normal distribution. IAP at each time point was analyzed as post values in the intervention group vs post values in the control group by ANCOVA. The $X^{2}$ test or Fisher exact test was performed for categorical variables, and relative risk (RR) was calculated for dichotomous variables. Two-tailed $P<0.05$ was considered statistically significant. The analyses were performed using the SPSS25.0 statistical software (IBM Corp, Armonk, NY).

\section{Results}

\section{Participant characteristics}

From 1 September 2015 to 15 August 2017, 552 AP patients admitted to Pancreatic ICU in our hospital were screened, of whom 185 patients with IAH were assessed for eligibility and 80 patients were included and randomized to neostigmine $(n=40)$ or conventional $(n=40)$ treatment (Fig. 1). The etiology of AP in $41(51 \%)$ patients was hypertriglyceridemia, defined as admission serum triglyceride level $>1000 \mathrm{mg} / \mathrm{dL}$ $(11.3 \mathrm{mmol} / \mathrm{L})$ and/or lipemic serum excluding other causes $[20,21]$, followed by $26(32.5 \%)$ biliary and 8 (10\%) alcohol excess. Before randomization, 38 (47.5\%) patients had respiratory failure and $48(60 \%)$ had IAP $>15 \mathrm{mmHg}$ (15 (18.8\%) with ACS). Four patients in the neostigmine group and 1 patient in the conventional group used opioids. In the neostigmine group, 3 patients had colonic ileus, 1 of whom used opioids.

Baseline characteristics were equally distributed between the two treatment groups (Table 2). There was no significant difference in baseline IAP between neostigmine and conventional groups $(16.3 \pm 2.7$ vs. $15.9 \pm 2.4, P=0.63)$. In the neostigmine group, there were $33(82.5 \%), 9(22.5 \%)$ and $23(57.5 \%)$ patients who received neostigmine every $12 \mathrm{~h}$, for 3 days or for 7 days, respectively (Additional file 1: Table S1). In the conventional group, 4 patients were eventually given neostigmine because of a continuous increase in IAP. Therefore, separate per-protocol analyses were also conducted after excluding the aforementioned 4 patients. The baseline parameters remained comparable between the two groups in the per-protocol analysis (Additional file 1: Table S2).

\section{Intra-abdominal pressure after randomization}

The IAP decreased after randomization in both groups, but dropped significantly faster as assessed at multiple time points in the neostigmine group than the conventional group (Table 3). IAP decreased from $16.3 \pm 2.7$ to $13.8 \pm 3.5 \mathrm{mmHg}$ after $9 \mathrm{~h}$ of neostigmine administration. IAP levels were significantly lower in neostigmine group than conventional treatment group at $9 \mathrm{~h}$ (13.8 \pm 3.5 vs. $15.0 \pm 3.1, P=0.038), 15 \mathrm{~h}(13.3 \pm 3.4$ vs. $14.7 \pm 3.1, P=0.015)$, and $168 \mathrm{~h}(12.2 \pm 2.7 \mathrm{vs}$. $13.6 \pm 3.5, P=0.045)$ after randomization.

Subgroup analysis restricting patient inclusion to baseline IAP $>15 \mathrm{mmHg}$ showed that the mean IAP levels in the neostigmine group were significantly lower than conventional group at $3 \mathrm{~h}, 9 \mathrm{~h}, 24 \mathrm{~h}, 30 \mathrm{~h}$, and $168 \mathrm{~h}$ after randomization (all $P \leq 0.038$ ) (Table 3). In per-protocol analysis, IAP at $15 \mathrm{~h}$ remained markedly lower in neostigmine group $(P=0.015)$ (Table 3$)$.

\section{Primary outcome}

Intension-to-treat analysis (Table 4) showed that the rate of decrease in IAP was significantly faster in the neostigmine group compared to the conventional group by $24 \mathrm{~h}$ (median with 25 th -75 th percentile: $-18.7 \%$ [ -28.4 to $-4.7 \%$ ) vs $-5.4 \%$ [ $-18.0 \%$ to 0$], P=0.017$ ). Intension-to-treat subgroup analysis of AP patients with baseline IAP $>15 \mathrm{mmHg}$ and per-protocol analysis also showed that IAP decreased significantly faster in the neostigmine group than in the conventional group (Additional file 1: Tables S3 and S4). 


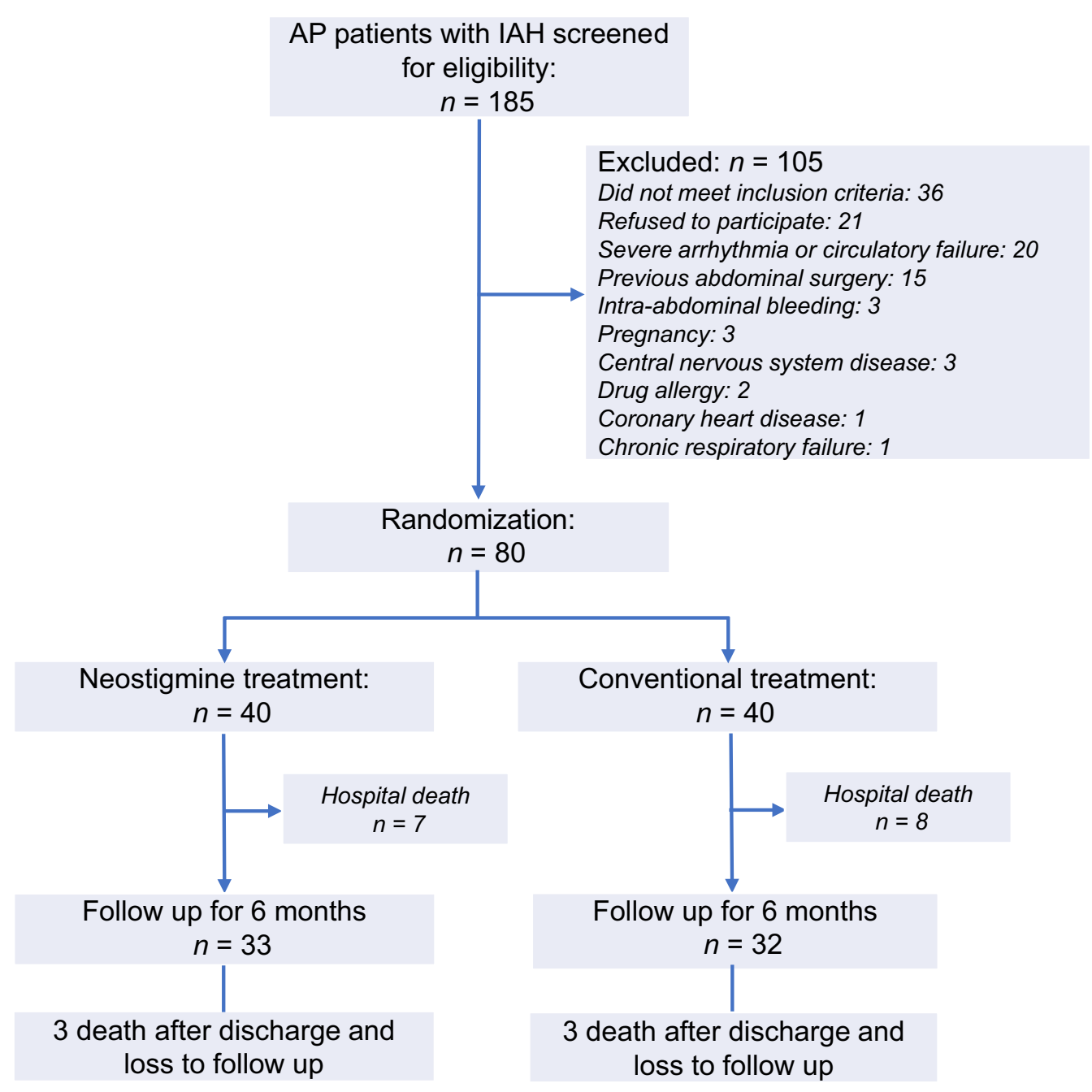

Fig. 1 Study flowchart. AP, acute pancreatitis; IAH, intra-abdominal hypertension

\section{Secondary outcomes}

Stool volumes (above baseline) were consistently higher in the neostigmine group compared to the conventional group from day 1 (median with 25th-75th percentile: $870 \mathrm{ml}$ [250-2070] vs. $60 \mathrm{ml}[30-770], P=0.00)$ to day 7 [1025 ml [450-1520] vs. $370 \mathrm{ml}[150-1200], P=0.02$; Fig. 2 and Table 4). The timing of EN (2 [0-3] vs. 2 [0-3] days), deterioration of IAH (4/40 vs. $8 / 40)$, new-onset ACS $(2 / 40$ vs $4 / 40)$, new-onset organ failure $(12 / 40$ vs. $16 / 40)$, mortality (7/40 vs. $8 / 40)$ and other outcome measures including follow-up for 6 months were not significantly different. These results remained unchanged by per-protocol analysis (Additional file 1: Table S4). In the subgroup analysis for baseline IAP $>15 \mathrm{mmHg}$, only stool volumes (above baseline) at day 1 were higher in the neostigmine group compared to the conventional group; all the remaining results were not statistically different between the two groups (Additional file 1: Table S3).
Among 12 patients with worsening IAH, 3 patients had intra-abdominal bleeding, 2 of whom underwent successful hemostasis and IAH subsequently decreased. Hemostasis could not be achieved in 1 patient who eventually died from uncontrollable progressive ACS. Five of the 12 patients with worsening IAH were fluid overloaded, so diuretics, albumin and/or renal replacement therapy were instituted: IAP reduced in 1, who survived; 2 developed ACS and multiple organ failure and died, while 2 had persistent respiratory failure, sepsis and died. The remaining 4 patients with worsening IAH had $>50 \%$ pancreatic necrosis and fluid accumulation in the retroperitoneum: 1 did not develop ACS or infection and survived, while 3 developed ACS, infected pancreatic necrosis, multiple organ failure and died. 
Table 2 Intention-to-treat analysis of baseline characteristics

\begin{tabular}{|c|c|c|c|}
\hline Characteristic & Neostigmine $(n=40)$ & Conventional $(n=40)$ & $P$ value \\
\hline Age (year) & $46 \pm 13$ & $49 \pm 14$ & 0.85 \\
\hline $\operatorname{Sex}(m / f)$ & $27 / 13$ & $34 / 6$ & 0.11 \\
\hline \multicolumn{4}{|l|}{ Etiology } \\
\hline Biliary & $12(30.0 \%)$ & $14(35.0 \%)$ & \multirow[t]{4}{*}{0.95} \\
\hline Hypertriglyceridemia $^{a}$ & $21(52.5 \%)$ & $20(50.0 \%)$ & \\
\hline Alcohol excess & $4(10.0 \%)$ & $4(10.0 \%)$ & \\
\hline Idiopathic & $3(7.5 \%)$ & $2(5.0 \%)$ & \\
\hline AP onset to hospital admission (d) & $3(1-4)$ & $2(1-3)$ & 0.06 \\
\hline AP onset to randomization (d) & $5(3-7)$ & $5(4-6)$ & 0.55 \\
\hline \multicolumn{4}{|l|}{ Comorbidity } \\
\hline Diabetes mellitus & $3(7.5 \%)$ & $6(15.0 \%)$ & 0.48 \\
\hline Hypertension & $2(5.0 \%)$ & $7(17.5 \%)$ & 0.15 \\
\hline Coronary heart disease & $1(2.5 \%)$ & 0 & 1.00 \\
\hline Chronic renal insufficiency & 0 & $1(2.5 \%)$ & 1.00 \\
\hline \multicolumn{4}{|l|}{ Admission clinical severity } \\
\hline SIRS & $2(2-3)$ & $2(2-3)$ & 0.70 \\
\hline APACHE ॥ & $9(7-9)$ & $9(7-12)$ & 0.79 \\
\hline C-reactive protein (mg/L) & $228.6 \pm 144.1$ & $295.8 \pm 125.8$ & 0.70 \\
\hline White cell count $\left(\times 10^{9} / \mathrm{L}\right)$ & $14.7 \pm 5.9$ & $14.2 \pm 5.6$ & 0.45 \\
\hline Procalcitonin $(\mathrm{ng} / \mathrm{mL})$ & $1.7(0.6-13.7)$ & $2.8(1.3-6.7)$ & 0.40 \\
\hline Serum lactate & $2.0 \pm 1.3$ & $1.7 \pm 0.9$ & 0.16 \\
\hline Organ failure & $32(80.0 \%)$ & $27(67.5 \%)$ & 0.31 \\
\hline \multicolumn{4}{|l|}{ Single organ failure } \\
\hline Respiratory & $21(52.5 \%)$ & $17(42.5 \%)$ & 0.50 \\
\hline Renal & $3(7.5 \%)$ & $1(2.5 \%)$ & 0.61 \\
\hline Multiple organ failure & $8(20.9 \%)$ & $9(22.5 \%)$ & 1.00 \\
\hline CTSI within 1 week of AP onset ${ }^{c}$ & $5(3-7)$ & $5(3-7)$ & 0.99 \\
\hline ANC & $28(73.7 \%)$ & $26(76.4 \%)$ & 0.63 \\
\hline APFC & $10(26.3 \%)$ & $8(23.5 \%)$ & 0.59 \\
\hline IAH level before randomization, $\mathrm{mmHg}$ & $16.3 \pm 2.7$ & $15.9 \pm 2.4$ & 0.63 \\
\hline Grade I & $15(37.5 \%)$ & $17(42.5 \%)$ & \\
\hline Grade II & $22(55.0 \%)$ & $21(52.5 \%)$ & \\
\hline Grade III & $3(7.5 \%)$ & $2(5.0 \%)$ & \\
\hline Grade IV & 0 & 0 & \\
\hline ACS & $9(22.5 \%)$ & $6(15.0 \%)$ & 0.56 \\
\hline Use of opioids & $4(10.0 \%)$ & $1(2.5 \%)$ & 0.36 \\
\hline Colonic ileus $s^{c, d}$ & $3(7.9 \%)$ & 0 & 0.11 \\
\hline $24 \mathrm{~h}$ of defecation $(\mathrm{mL})$ & $450(10-1050)$ & $800(520-990)$ & 0.14 \\
\hline PCD of ascites & $10(25.0 \%)$ & $6(15.0 \%)$ & 0.40 \\
\hline Admitted to the ICU at randomization & $40(100 \%)$ & $40(100 \%)$ & 1.00 \\
\hline
\end{tabular}

ACS abdominal compartment syndrome, AP acute pancreatitis, APACHE /l acute physiology and chronic health evaluation II, APFC acute peripancreatic fluid collection, $A N C$ acute necrotic collection, CTSI computed tomography severity index, IAH intra-abdominal hypertension, ICU Intensive Care Unit, $P C D$ percutaneous catheter drainage, RAC Revised Atlanta Classification, SAP severe acute pancreatitis, SIRS systemic inflammatory response syndrome

${ }^{a}$ Defined as admission serum triglyceride level $>1000 \mathrm{mg} / \mathrm{dL}$ and/or lipemic serum after ruling out biliary and alcohol excess etiologies

${ }^{b}$ Patients with circulatory failure were excluded because neostigmine may affect the circulation

'There were 38 and 34 cases in the neostigmine group and conventional group, respectively, underwent CT within the first week after AP onset

d Opioids were used in 2 of the 3 patients with colonic ileus 
Table 3 Intra-abdominal pressure from randomization to 7 days

\begin{tabular}{|c|c|c|c|c|c|c|c|c|c|}
\hline \multirow[t]{3}{*}{ Time (h) } & \multicolumn{3}{|c|}{ Intention-to-treat analysis } & \multicolumn{3}{|c|}{$\begin{array}{l}\text { Subgroup analysis (IAP > } 15 \mathrm{mmHg} \text { at } \\
\text { baseline) }\end{array}$} & \multicolumn{3}{|c|}{ Per-protocol analysis } \\
\hline & $\begin{array}{l}\text { Neostigmine } \\
(n=40)\end{array}$ & $\begin{array}{l}\text { Conventional } \\
(n=40)\end{array}$ & $P$ value $^{\dagger}$ & $\begin{array}{l}\text { Neostigmine } \\
(n=25)\end{array}$ & $\begin{array}{l}\text { Conventional } \\
(n=23)\end{array}$ & $P$ value $^{\dagger}$ & $\begin{array}{l}\text { Neostigmine } \\
(n=40)\end{array}$ & $\begin{array}{l}\text { Conventional } \\
(n=36)\end{array}$ & $P$ value $^{\dagger}$ \\
\hline & IAP & IAP & & IAP & IAP & & IAP & IAP & \\
\hline 0 & $16.3 \pm 2.7$ & $15.9 \pm 2.4$ & & $17.9 \pm 2.0$ & $17.6 \pm 1.7$ & & $16.3 \pm 2.7$ & $15.9 \pm 2.5$ & \\
\hline 3 & $14.6 \pm 3.0$ & $15.0 \pm 3.1$ & 0.205 & $15.0 \pm 3.0$ & $16.7 \pm 2.6$ & 0.010 & $14.6 \pm 3.0$ & $14.9 \pm 2.9$ & 0.322 \\
\hline 9 & $13.8 \pm 3.5$ & $15.0 \pm 3.1$ & 0.038 & $14.2 \pm 3.3$ & $16.0 \pm 3.4$ & 0.018 & $13.8 \pm 3.5$ & $14.7 \pm 2.8$ & 0.079 \\
\hline 15 & $13.3 \pm 3.4$ & $14.7 \pm 3.1$ & 0.015 & $13.2 \pm 3.7$ & $15.8 \pm 3.0$ & 0.001 & $13.3 \pm 3.4$ & $14.7 \pm 3.1$ & 0.015 \\
\hline 24 & $13.7 \pm 3.6$ & $14.7 \pm 3.2$ & 0.083 & $13.7 \pm 3.6$ & $15.2 \pm 3.2$ & 0.020 & $13.7 \pm 3.6$ & $14.5 \pm 3.1$ & 0.152 \\
\hline 30 & $13.7 \pm 3.5$ & $14.3 \pm 3.1$ & 0.323 & $13.4 \pm 3.2$ & $15.0 \pm 2.9$ & 0.038 & $13.7 \pm 3.5$ & $14.0 \pm 3.1$ & 0.533 \\
\hline 36 & $14.3 \pm 3.6$ & $13.8 \pm 3.0$ & 0.619 & $14.2 \pm 3.5$ & $14.1 \pm 3.1$ & 0.849 & $14.3 \pm 3.6$ & $13.7 \pm 3.0$ & 0.521 \\
\hline 42 & $13.8 \pm 3.1$ & $14.2 \pm 2.6$ & 0.454 & $13.7 \pm 3.4$ & $14.5 \pm 2.4$ & 0.183 & $13.8 \pm 3.1$ & $14.2 \pm 2.5$ & 0.465 \\
\hline 48 & $14.1 \pm 3.2$ & $13.7 \pm 2.9$ & 0.767 & $14.8 \pm 3.0$ & $14.4 \pm 2.2$ & 0.895 & $14.1 \pm 3.2$ & $13.4 \pm 2.9$ & 0.489 \\
\hline 54 & $13.6 \pm 3.4$ & $13.4 \pm 2.8$ & 0.961 & $14.1 \pm 3.4$ & $14.0 \pm 2.1$ & 0.845 & $13.6 \pm 3.4$ & $13.2 \pm 2.6$ & 0.790 \\
\hline 60 & $13.0 \pm 3.1$ & $13.6 \pm 2.6$ & 0.280 & $13.0 \pm 3.2$ & $14.1 \pm 2.1$ & 0.097 & $13.0 \pm 3.1$ & $13.3 \pm 2.4$ & 0.522 \\
\hline 66 & $13.6 \pm 3.1$ & $13.3 \pm 3.9$ & 0.853 & $14.0 \pm 2.8$ & $13.7 \pm 3.4$ & 0.904 & $13.6 \pm 3.1$ & $12.9 \pm 3.7$ & 0.468 \\
\hline 72 & $13.2 \pm 2.9$ & $13.8 \pm 2.9$ & 0.237 & $13.8 \pm 3.1$ & $14.2 \pm 2.4$ & 0.490 & $13.2 \pm 2.9$ & $13.4 \pm 2.6$ & 0.589 \\
\hline 120 & $13.2 \pm 3.2$ & $13.8 \pm 2.9$ & 0.213 & $13.6 \pm 3.2$ & $14.4 \pm 2.8$ & 0.261 & $13.2 \pm 3.2$ & $13.2 \pm 2.2$ & 0.731 \\
\hline 168 & $12.2 \pm 2.7$ & $13.6 \pm 3.5$ & 0.045 & $11.9 \pm 2.8$ & $14.2 \pm 3.6$ & 0.013 & $12.2 \pm 2.7$ & $13.0 \pm 2.7$ & 0.199 \\
\hline
\end{tabular}

IAP, intra-abdominal pressure

${ }^{+}$IAP at each time point were analyzed as post values in the intervention group versus post values in the control group by ANCOVA

\section{Adverse events}

Neostigmine treatment was considered unlikely to be related to all 6 adverse events (Additional file 1: Tables S5 and S6). The new-onset circulatory failure occurred in 3 patients in the neostigmine group and 1 patient in the conventional group given neostigmine because of continuous increase in IAP. All 4 had respiratory and/or renal failure prior to randomization, and circulatory failure was attributed to progression of AP. One patient in the neostigmine group developed new-onset respiratory failure after rebound elevation in IAP leading to ACS. Overall, 12 patients in the neostigmine group compared to 16 patients in the conventional group developed newonset organ failure after randomization (new-onset organ failure after cessation or in the absence of neostigmine administration was not recorded as an adverse event). One patient in the neostigmine group developed bradycardia while also receiving esmolol, a cardio-selective beta ${ }_{1}$ receptor blocker with rapid onset and short duration of action; the bradycardia ceased after withdrawal of esmolol during continuation of neostigmine.

\section{Discussion}

This first randomized controlled trial of neostigmine as a promotility agent for AP patients with IAH/ACS showed neostigmine treatment was significantly more effective than conventional treatment in reducing IAP in AP patients with persistent IAH after $24 \mathrm{~h}$ of conventional treatment (including gastrointestinal decompression, glycerin enema, negative fluid balance, and ascites drainage). The decrease in IAP occurred within $3 \mathrm{~h}$ of neostigmine administration and was most pronounced within the first $15 \mathrm{~h}$ and more evident in patients with severe IAP ( $>15 \mathrm{mmHg}$ ) at baseline. The reduction in IAP appears relatively modest in neostigmine group; the first reason is that the patients included in this study were difficult to reduce the IAP to normal after $24 \mathrm{~h}$ of other simple measures; secondly, for ethical reasons, patients in the conventional group also used Chinese traditional medicine-rheum officinale and glauber salt to promote gastrointestinal peristalsis after randomization. A small pilot study on SAP patients found that dachengqi decoction with rheum officinale and glauber salt as the main components can significantly reduce IAP on days 4-8 after admission [11]. We believe that the use of neostigmine in patients with IAH above grade II $(>15 \mathrm{mmHg})$ is more clinically significant, since the effect of neostigmine on reducing IAP is more profound in patients with IAP greater than $15 \mathrm{mmHg}$. Subclinical organ injury develops at levels of IAP previously deemed to be safe (IAP between 12 and $15 \mathrm{mmHg}$ ), but as IAP increases, organ dysfunction will become more pronounced, and there is a dose-dependent relationship between IAP and organ dysfunction [22]. 
Table 4 Intention-to-treat analysis of primary endpoint and secondary endpoints

\begin{tabular}{|c|c|c|c|c|}
\hline Endpoint & Neostigmine $(n=40)$ & Conventional $(n=40)$ & RR $(95 \% \mathrm{Cl})$ & $P$ value \\
\hline \multicolumn{5}{|l|}{ Primary endpoint } \\
\hline Percent change of IAP at $24 \mathrm{~h}, \%$ & $-18.7([-28.4]-[-4.7])$ & $-5.4([-18.0]-0)$ & & 0.017 \\
\hline \multicolumn{5}{|l|}{ Secondaryendpoint } \\
\hline Increase in stool volume at $24 \mathrm{~h}$ after randomization $(\mathrm{mL})$ & $870(250-2070)$ & $60([-30]-770)$ & & 0.00 \\
\hline Increase in stool volume at $7 \mathrm{~d}$ after randomization $(\mathrm{mL})$ & $1025(450-1520)$ & $370(150-1200)$ & & 0.02 \\
\hline Serum lactate & $1.7 \pm 0.8$ & $1.6 \pm 0.7$ & & 0.97 \\
\hline Timing of EN ${ }^{a}$ & $2(0-3)$ & $2(0-3)$ & & 1.00 \\
\hline Deterioration of $\mathrm{IAH}$ & $4(10.0 \%)$ & $8(20.0 \%)$ & $0.50(0.16-1.53)$ & 0.35 \\
\hline New-onset ACS & $2(5.0 \%)$ & $4(10.0 \%)$ & $0.50(0.10-2.58)$ & 0.68 \\
\hline New-onset organ failure & $12(30.0 \%)$ & $16(40.0 \%)$ & $0.75(0.41-1.38)$ & 0.48 \\
\hline \multicolumn{5}{|l|}{ Single organ failure } \\
\hline Respiratory & $2(5.0 \%)$ & $6(15.0 \%)$ & $0.33(0.07-1.55)$ & 0.26 \\
\hline Circulatory & $3(7.5 \%)$ & $3(7.5 \%)$ & $1.00(0.21-4.66)$ & 1.00 \\
\hline Renal & 0 & $3(7.5 \%)$ & - & 0.24 \\
\hline Multiple organ failure & $7(15.0 \%)$ & $4(10.0 \%)$ & $1.75(0.56-5.52)$ & 0.52 \\
\hline \multicolumn{5}{|l|}{ Invasive interventions $^{c}$} \\
\hline Percutaneous catheter drainage & $8(20.0 \%)$ & $5(12.5 \%)$ & $1.60(0.58-4.48)$ & 0.55 \\
\hline Endoscopic transmural drainage & $3(7.5 \%)$ & $4(10.0 \%)$ & $0.75(0.18-3.04)$ & 1.00 \\
\hline Endoscopic necrosectomy ${ }^{d}$ & $1(2.5 \%)$ & $2(5.0 \%)$ & $0.50(0.05-5.30)$ & 1.00 \\
\hline Surgical laparotomy & $3(7.5 \%)$ & $4(10.0 \%)$ & $0.75(0.18-3.14)$ & 1.00 \\
\hline Intra-abdominal bleeding (requiring intervention) & $2(5.0 \%)$ & $4(10.0 \%)$ & $0.50(0.10-2.58)$ & 0.68 \\
\hline Enterocutaneous fistula (requiring intervention) & $2(5.0 \%)$ & 0 & - & 0.49 \\
\hline Septicemia & $11(27.5 \%)$ & $11(27.5 \%)$ & $1.00(0.49-2.04)$ & 1.00 \\
\hline Vascular complications ${ }^{e}$ & $4(11.8 \%)$ & $5(13.5 \%)$ & $0.87(0.26-2.98)$ & 0.56 \\
\hline Portal veinthrombosis & 0 & $3(7.5 \%)$ & & 0.24 \\
\hline Splenic vein thrombosis / splenic infarction & $3(7.5 \%)$ & $1(2.5 \%)$ & & 0.53 \\
\hline Portal vein and splenic vein thrombosis & 0 & $1(2.5 \%)$ & & 0.49 \\
\hline Superior mesenteric vein and splenic vein thrombosis & $1(2.5 \%)$ & 0 & & 0.49 \\
\hline \multicolumn{5}{|l|}{ RAC disease severity } \\
\hline MSAP & $3(7.5 \%)$ & $5(12.5 \%)$ & $0.60(0.15-2.34)$ & 0.71 \\
\hline SAP & $37(92.5 \%)$ & $35(87.5 \%)$ & $1.05(0.91-1.22)$ & 0.71 \\
\hline Death in index hospital stay & $7(17.5 \%)$ & $8(20 \%)$ & $0.88(0.35-2.18)$ & 0.77 \\
\hline Length of ICU stay (d) & $14 \pm 9$ & $15 \pm 14$ & & 0.94 \\
\hline Length of hospital stay (d) & $23 \pm 13$ & $22 \pm 16$ & & 0.48 \\
\hline Medical expenses (1000 RMB) & $125.9 \pm 83.6$ & $134.3 \pm 128.8$ & & 0.80 \\
\hline Follow-up (6 M) & $N=33$ & $N=32$ & & \\
\hline Pancreatic pseudocyst & $2(6.1 \%)$ & $1(3.1 \%)$ & $1.94(0.18-20.35)$ & 1.00 \\
\hline Needing elective intervention & 0 & $1(3.1 \%)$ & - & 0.49 \\
\hline Walled-off necrosis & $14(42.4 \%)$ & $11(34.4 \%)$ & $1.23(0.66-2.30)$ & 0.61 \\
\hline Needing elective intervention & $3(9.1 \%)$ & $1(3.1 \%)$ & $2.91(0.32-26.52)$ & 0.61 \\
\hline Portal thrombosis & $1(3.1 \%)$ & $1(3.1 \%)$ & $0.97(0.06-14.85)$ & 1.00 \\
\hline Pancreatogenic portal hypertension & $1(3.1 \%)$ & $2(6.1 \%)$ & $0.48(0.04-5.62)$ & 1.00 \\
\hline New onset diabetes & $9(27.3 \%)$ & $5(15.6 \%)$ & $2.03(0.60-6.88)$ & 0.37 \\
\hline Impaired glucose tolerance & $3(9.1 \%)$ & $2(6.3 \%)$ & $1.55(0.23-19.63)$ & 1.00 \\
\hline External secretion dysfunction & $7(24.1 \%)$ & $4(13.3 \%)$ & $2.06(0.53-8.00)$ & 0.33 \\
\hline Recurrent AP & $4(12.2 \%)$ & $1(3.1 \%)$ & $4.28(0.45-40.53)$ & 0.36 \\
\hline Death after discharge & $3(9.1 \%)$ & $3(9.4 \%)$ & $0.97(0.18-5.19)$ & 1.00 \\
\hline
\end{tabular}

ACS abdominal compartment syndrome, $A P$ acute pancreatitis, $C l$ confidence interval, $E N$ enteral nutrition, IAH intra-abdominal hypertension, ICU Intensive Care Unit, $M S A P$ moderately severe acute pancreatitis, $R R$ relative risk, $S A P$ severe acute pancreatitis

a Time from randomization to initiation of EN

${ }^{\mathrm{b}}$ IAP that rebounded $\geq 5 \mathrm{mmHg}$ or increased $\geq 20 \mathrm{mmHg}$ in $1-7$ days after randomization 
Table 4 (continued)

\footnotetext{
c All interventions after randomization were counted

${ }^{d}$ In the neostigmine group, 1 case underwent endoscopic debridement; in the conventional group, 1 case underwent percutaneous retroperitoneal endoscopic debridement and 1 case underwent endoscopic debridement

e 30 cases in the neostigmine group, and 32 cases in conventional group received CT enhancement and vascular imaging
}

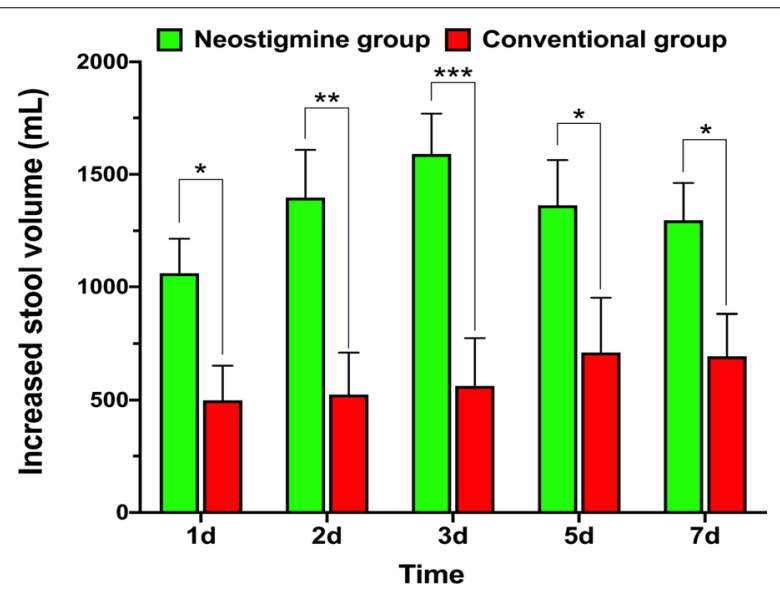

Fig. 2 Intention-to-treat plots of increased stool volumes $(\mathrm{mL} / 24 \mathrm{~h}$ minus baseline $24 \mathrm{~h}$ stool volume before randomization). ${ }^{*} P<0.05$, $\left.{ }^{* *} P<0.01,{ }^{* * *} P<0.001\right)$

As IAH/ACS is associated with a higher incidence of organ dysfunction [23], higher mortality and longer hospitalization in patients with SAP [24], reducing IAP as promptly as possible may improve outcomes in AP. We found no statistically significant difference, however, in the incidence of new organ failure or other complications between neostigmine and conventional treatment, although patients receiving neostigmine showed a trend toward less new-onset respiratory ( 2 of 40 vs. 6 of 40), renal (0 vs. 3 ) and overall organ (12 vs. 16) failure.

Neostigmine treatment resulted in significantly increased stool volumes compared to conventional treatment, confirming enhanced intestinal peristalsis, although this did not significantly hasten the initiation of EN. Early EN has been shown to enhance recovery in AP, likely by protecting the gut mucosal barrier and reducing bacterial translocation, infected pancreatic necrosis and other severe outcomes [25]. The optimal time to initiate EN in patients with AP is considered to be within 24-72 $\mathrm{h}$ of admission, allowing for early intolerance to oral feeding [26]. IAH/ACS impedes early EN and is commonly associated with food intolerance. A prospective pilot study found early EN to hinder the development of IAH and reduce the severity of SAP compared with delayed EN [17]. In our study, neostigmine treatment promoted gut motility and thus reduced IAP, which may increase tolerance to EN. The median time to initiation of EN in the neostigmine and conventional groups was, however, comparable at the second day after randomization (third day after admission to Pancreatic ICU), within the period recommended in guidelines [27]. European Society of Intensive Care Medicine (ESICM) clinical practice guidelines recommend EN should be administered with caution when IAP reaches $\geq 15 \mathrm{mmHg}$ [27]. The high proportion (48, i.e., 60\%) of our patients who had IAP $\geq 15 \mathrm{mmHg}$ at entry into a trial may partially explain the lack of any significant effect of neostigmine on the timing of EN initiation.

Neostigmine blocks the active site of anticholinesterase, increasing the availability of acetylcholine to ligate nicotinic ion channel receptors and muscarinic G-protein receptors that elicit second messenger cascades [28]. Muscarinic receptors predominate in the enteric nervous system, which response to vagal parasympathetic preganglionic activation, as well as operating independently to drive motility, secretion, the cholinergic anti-inflammatory pathway, epithelial proliferation and repair [28, 29]. Vagal nerve stimulation has shown consistent antiinflammatory effects in colitis models, whereas vagotomy increases inflammatory markers in these models [28] and greater severity of pancreatic injury in experimental AP [30]. The evidence from our trial indicates a direct beneficial effect of neostigmine on the gut function that may include reduction in gut injury, which might contribute to improved outcomes from AP. Despite the predominance of hypertriglyceridemia etiology in our trial (41 of 80 patients), which has a high incidence in China [31-34], and induces more SAP [20, 34, 35], the mechanisms of action of neostigmine are likely to apply equally to other etiologies of human AP, although this remains to be tested further.

Patients in both the neostigmine and conventional groups were given rheum officinale (rhubarb) and glauber salt to promote defecation. Rhubarb and glauber salt are principal constituents of dachengqi decoction and its derivatives, commonly used in China to treat AP $[15,16]$, but they are rarely used in other countries in the world. Modified dachengqi decoction significantly decreased IAP 4-8 days after admission and improved clinical outcomes in a randomized trial conducted in predicted SAP patients [36]. A recent meta-analysis [37] of 11 randomized trials has shown that the combination of rhubarb and early EN compared to early EN alone improves gut motility, enhances the efficacy of EN and reduces AP severity in predicted SAP patients. Despite the use of 
rhubarb and glauber salt in both our treatment groups; however, neostigmine had significant beneficial effects on IAP and gut function, indicative of an independent action that may be widely applicable to patients with AP and $\mathrm{IAH} \pm \mathrm{ACS}$.

Neostigmine was not effective in reducing IAH in all AP patients. In the neostigmine group, IAP rebounded by $\geq 5 \mathrm{mmHg}$ or rose to $>20 \mathrm{mmHg}$ in 4 patients, 2 of whom developed new ACS. In addition to ileus, distention, inflammation and gut wall edema, extra-luminal factors may contribute to the development of IAH in AP. These factors include third space fluid losses, acute fluid collections, fluid overload, pancreatic/peri-pancreatic necrosis and/or intra-abdominal hemorrhage, each of which may contribute to the failure of treatment for IAH, notwithstanding additional water losses in stool from neostigmine treatment. A previous randomized controlled trial found controlled fluid resuscitation reduced the incidence of ACS in patients with SAP [38], although optimal protocols are yet to be worked out.

In this study, we did not observe any adverse event likely to be related to neostigmine treatment, but a potential safety signal remains, since neostigmine may increase cardiac output by lowering IAP and thus systemic vascular resistance, yet result in an increased cholinergic drive that may slow heart rate [13]. Symptomatic bradycardia has been observed in patients treated with neostigmine for acute colonic pseudo-obstruction, but the bradycardia that developed in our patient resolved on cessation of the $\beta$-adrenergic antagonist esmolol. Patients with underlying brady-arrhythmias or those receiving $\beta$-adrenergic antagonists are likely to be more susceptible to bradycardia during the treatment of neostigmine [10]. In addition to bradycardia, neostigmine may also cause bronchoconstriction and increase airway resistance [13], although this has not been observed in previous studies [10-13] and was not in our study. A further caution would be the late use of neostigmine 4-6 weeks after the onset of AP, because of a risk of acute or sub-acute intestinal obstruction in the presence of adhesions caused by fibrosis of necrotic tissue.

\section{Limitations}

This trial was not blinded, allowing for flexibility to alter the frequency of neostigmine administration depending on changes in IAP, but introducing potential bias, e.g., timing the start of EN. As neostigmine treatment every $12 \mathrm{~h}$ was sufficient for the vast majority of (35 of 40) patients, this frequency or specific rules could be adopted in any future double-blind trial. Our trial had a relatively small sample size, designed as a phase II trial to test the efficacy of neostigmine on the reduction in IAP, but not on the complications of AP.

\section{Conclusion}

This preliminary study found neostigmine to reduce IAP effectively in patients with AP and IAH who were not responding to conventional treatment, by enhancing intestinal peristalsis and promoting defecation, especially in patients with baseline IAP $>15 \mathrm{mmHg}$. These results warrant a larger, multi-center, phase III trial designed to assess the impact of neostigmine on the complications of AP from multiple etiologies.

\section{Abbreviations \\ ACS: Abdominal compartment syndrome; AP: Acute pancreatitis; CT: Com- puted tomography; EN: Enteral nutrition; IAH: Intra-abdominal hypertension; IAP: Intra-abdominal pressure; PCD: Percutaneous catheter drainage; SAP: Severe acute pancreatitis.}

\section{Supplementary Information}

The online version contains supplementary material available at https://doi. org/10.1186/s13054-022-03922-4.

Additional file 1: Table S1. Schedule of neostigmine administration in the neostigmine group: (a) frequency and (b) duration, Table S2. Per-protocol analysis of baseline characteristics, Table S3. Per-protocol analysis of secondary endpoints, Table S4. Subgroup analysis of secondary endpoints (IAP $\geq 15 \mathrm{mmHg}$ at randomization), Table S5. Characteristics of patients who developed adverse events, Table S6. Adverse events, causes and outcomes.

\section{Acknowledgements}

We would like to thank all of the doctors, nurses, technicians and patients involved at the participating centers for their dedication to the study.

\section{Authors' contributions}

WHE, YZ and NL designed the research. $L X, Y Z, P L, H Z, Y W, H K$ and $X H$ performed the research. $P C, Y L$, and $Y Z$ collected data. WHE, $X S$, and $W C$ analyzed the data. WHE, PC, YL and WC drafted of the manuscript. WHU, RS, YZ, and NL reviewed and revised the manuscript. All authors read and approved the final manuscript.

\section{Funding}

National Key Clinical Specialist Project [No. (2011)872]; National Natural Science Foundation of China (No. 81660114, No. 81860122); and Jiangxi Province Outstanding Youth Talent Funding Program (No. 20192BCBL23021); and Science and Technology Research Project of Jiangxi Provincial Department of Education (No. 60125). RS is supported by an NIHR Senior Investigator award. The funder of the study had no role in study design, data collection, data analysis, data interpretation, or writing of the report.

\section{Availability of data and materials}

The datasets used and/or analyzed during the current study are available from the corresponding author on reasonable request.

\section{Declarations}

Ethics approval and consent to participate

This trial was registered (ClinicalTrials.gov, No. NCT02543658) and conducted adhering to a protocol that was approved by the Medical Ethics Research Committee of our hospital (2015, No. 011). Written informed consent was obtained from a relative prior to inclusion.

Consent for publication

Not applicable. 


\section{Competing interests}

Robert Sutton has received research support and/or funding from Calcimedica, Cypralis, GlaxoSmithKline, MSD/Merck, and Novartis, has been a consultant for AbbVie, Calcimedica, Cypralis, Eagle Pharmaceuticals, Novartis, and Takeda (all funds to the University of Liverpool), and is collaborating in the IMI2 TransBioLine project with multiple public and private institutions including Janssen, Lilly, MSD/Merck, Novartis, Pfizer, Roche, and Sanofi-Aventis. The other authors declare no conflicts of interest.

\section{Author details}

${ }^{1}$ Pancreatic Intensive Care Unit, Department of Gastroenterology, First Affiliated Hospital of Nanchang University, Nanchang, China. ${ }^{2}$ Liverpool Pancreatitis Research Group, Institute of Systems, Molecular and Integrative Biology, University of Liverpool and Liverpool University Hospitals NHS Foundation Trust, Liverpool, Merseyside, UK. ${ }^{3}$ Departments of Integrated Traditional Chinese and Western Medicine \& Clinical Research Management, Sichuan Provincial Pancreatitis Center \& West China-Liverpool Biomedical Research Center, West China Hospital, Sichuan University, Chengdu, China. ${ }^{4}$ Chinese Evidence-Based Medicine Center and CREAT Group, West China Hospital, Sichuan University, Chengdu, China.

Received: 4 November 2021 Accepted: 30 January 2022 Published online: 03 March 2022

\section{References}

1. Mederos MA, Reber HA, Girgis MD. Acute pancreatitis: a review. JAMA. 2021;325:382-90.

2. Guo Q, Li A, Xia Q, et al. The role of organ failure and infection in necrotizing pancreatitis: a prospective study. Ann Surg. 2014;259:1201-7.

3. Schepers NJ, Bakker OJ, Besselink MG, et al. Impact of characteristics of organ failure and infected necrosis on mortality in necrotising pancreatitis. Gut. 2019;68:1044-51.

4. Garg PK, Singh VP. Organ failure due to systemic injury in acute pancreatitis. Gastroenterology. 2019;156:2008-23.

5. Shi N, Liu T, de la Iglesia-Garcia D, et al. Duration of organ failure impacts mortality in acute pancreatitis. Gut. 2020;69:604-5.

6. Kirkpatrick AW, Roberts DJ, De Waele J, et al. Intra-abdominal hypertension and the abdominal compartment syndrome: updated consensus definitions and clinical practice guidelines from the World Society of the Abdominal Compartment Syndrome. Intensive Care Med. 2013;39:1190-206.

7. Trikudanathan G, Vege SS. Current concepts of the role of abdominal compartment syndrome in acute pancreatitis: an opportunity or merely an epiphenomenon. Pancreatology. 2014;14:238-43.

8. Aitken EL, Gough V, Jones A, et al. Observational study of intra-abdominal pressure monitoring in acute pancreatitis. Surgery. 2014;155:910-8.

9. van Brunschot S, Schut AJ, Bouwense SA, et al. Abdominal compartment syndrome in acute pancreatitis: a systematic review. Pancreas. 2014:43:665-74.

10. Ponec RJ, Saunders MD, Kimmey MB. Neostigmine for the treatment of acute colonic pseudo-obstruction. N Engl J Med. 1999;341:137-41.

11. Amaro R, Rogers Al. Neostigmine infusion: new standard of care for acute colonic pseudo-obstruction? Am J Gastroenterol. 2000;95:304-5.

12. Loftus CG, Harewood GC, Baron TH. Assessment of predictors of response to neostigmine for acute colonic pseudo-obstruction. Am J Gastroenterol. 2002;97:3118-22.

13. Korsten MA, Rosman AS, Ng A, et al. Infusion of neostigmine-glycopyrrolate for bowel evacuation in persons with spinal cord injury. Am J Gastroenterol. 2005;100:1560-5.

14. Mehta R, John A, Nair P, et al. Factors predicting successful outcome following neostigmine therapy in acute colonic pseudo-obstruction: a prospective study. J Gastroenterol Hepatol. 2006;21:459-61.

15. Lu X, Xiao W, Kang X, et al. The effect of Chinese herbal medicine on non-biliogenic severe acute pancreatitis: a systematic review and metaanalysis. J Ethnopharmacol. 2014;155:21-9.

16. Qiong $W$, Yiping $W$, Jinlin $Y$, et al. Chinese medicinal herbs for acute pancreatitis. Cochrane Database Syst Rev. 2005;2005(1):CD003631. https:// doi.org/10.1002/14651858.CD003631.pub2.
17. Sun JK, LiWQ, Ke L, et al. Early enteral nutrition prevents intra-abdominal hypertension and reduces the severity of severe acute pancreatitis compared with delayed enteral nutrition: a prospective pilot study. World J Surg. 2013;37:2053-60.

18. Mirtallo JM, Forbes A, McClave SA, et al. International consensus guidelines for nutrition therapy in pancreatitis. JPEN J Parenter Enteral Nutr. 2012;36:284-91.

19. Pedersen SB, Langsted A, Nordestgaard BG. Nonfasting mild-to-moderate hypertriglyceridemia and risk of acute pancreatitis. JAMA Intern Med. 2016;176:1834-42.

20. Nawaz H, Koutroumpakis E, Easler J, et al. Elevated serum triglycerides are independently associated with persistent organ failure in acute pancreatitis. Am J Gastroenterol. 2015;110:1497-503.

21. Adiamah A, Psaltis E, Crook M, et al. A systematic review of the epidemiology, pathophysiology and current management of hyperlipidaemic pancreatitis. Clin Nutr. 2018;37:1810-22.

22. De Laet IE, Malbrain M, De Waele JJ. A clinician's guide to management of intra-abdominal hypertension and abdominal compartment syndrome in critically III patients. Crit Care. 2020;24(1):97.

23. Al-Bahrani AZ, Abid GH, Holt A, et al. Clinical relevance of intra-abdominal hypertension in patients with severe acute pancreatitis. Pancreas. 2008;36:39-43.

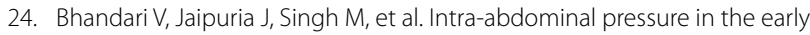
phase of severe acute pancreatitis: canary in a coal mine? Results from a rigorous validation protocol. Gut Liver. 2013;7:731-8.

25. Crockett SD, Wani S, Gardner TB, et al. American Gastroenterological Association Institute guideline on initial management of acute pancreatitis. Gastroenterology. 2018;154:1096-110.

26. Arvanitakis $M$, Ockenga J, Bezmarevic $M$, et al. ESPEN guideline on clinical nutrition in acute and chronic pancreatitis. Clin Nutr. 2020;39:612-31.

27. Reintam Blaser A, Starkopf J, Alhazzani W, et al. Early enteral nutrition in critically ill patients: ESICM clinical practice guidelines. Intensive Care Med. 2017:43:380-98.

28. Vaknine S, Soreq H. Central and peripheral anti-inflammatory effects of acetylcholinesterase inhibitors. Neuropharmacology. 2020;168:108020.

29. Brinkman DJ, Ten Hove AS, Vervoordeldonk MJ, et al. Neuroimmune interactions in the gut and their significance for intestinal immunity. Cells. 2019:8(7):670. https://doi.org/10.3390/cells8070670.

30. van Westerloo DJ, Giebelen IA, Florquin S, et al. The vagus nerve and nicotinic receptors modulate experimental pancreatitis severity in mice. Gastroenterology. 2006;130:1822-30.

31. Zhang $R$, Deng $L$, Jin $T$, et al. Hypertriglyceridaemia-associated acute pancreatitis: diagnosis and impact on severity. HPB (Oxford). 2019;21:1240-9.

32. Ding $Y$, Zhang $M$, Wang $L$, et al. Association of the hypertriglyceridemic waist phenotype and severity of acute pancreatitis. Lipids Health Dis. 2019;18:93.

33. Zheng $X$, Li L, Zhu Y, et al. Superoxide dismutase predicts persistent circulation failure and mortality in the early stage of acute pancreatitis. Dig Dis Sci. 2020:65:3551-7.

34. Wan J, He W, Zhu Y, et al. Stratified analysis and clinical significance of elevated serum triglyceride levels in early acute pancreatitis: a retrospective study. Lipids Health Dis. 2017;16:124.

35. Pascual I, Sanahuja A, Garcia N, et al. Association of elevated serum triglyceride levels with a more severe course of acute pancreatitis: cohort analysis of 1457 patients. Pancreatology. 2019;19:623-9.

36. Wan MH, Li J, Huang W, et al. Modified Da-Cheng-Qi decoction reduces intra-abdominal hypertension in severe acute pancreatitis: a pilot study. Chin Med J (Engl). 2012;125:1941-4.

37. Chen $X$, Yang $K$, Jing $G$, et al. Meta-analysis of efficacy of rhubarb combined with early enteral nutrition for the treatment of severe acute pancreatitis. JPEN J Parenter Enteral Nutr. 2020;44:1066-78.

38. Mao EQ, Tang YQ, Fei J, et al. Fluid therapy for severe acute pancreatitis in acute response stage. Chin Med J (Engl). 2009;122:169-73.

\section{Publisher's Note}

Springer Nature remains neutral with regard to jurisdictional claims in published maps and institutional affiliations. 